
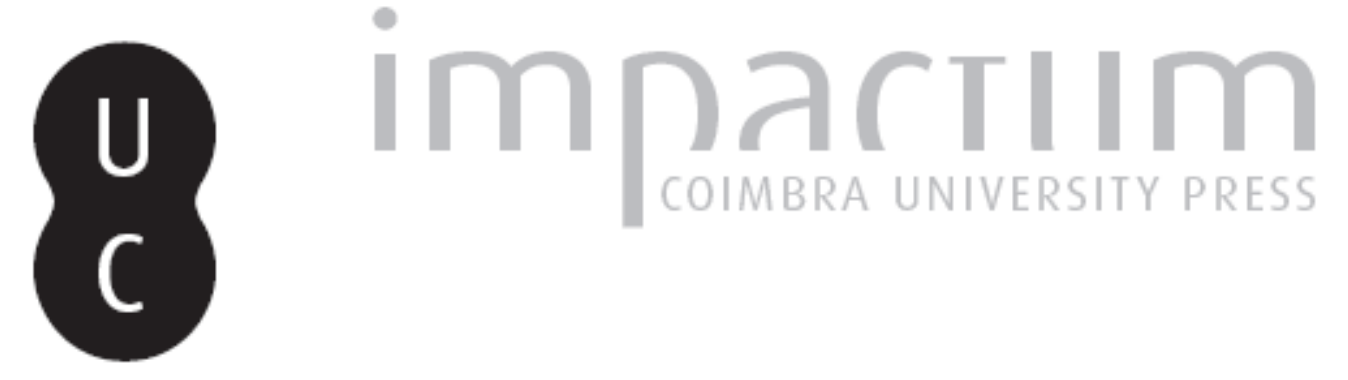

\title{
Fatores de controlo sobre a localização de grandes complexos de ravinas: uma análise com base em reconstrução topográfica e regressão logística
}

Autor(es): $\quad$ Bergonse, Rafaello; Reis, Eusébio

Publicado por: Imprensa da Universidade de Coimbra

URL persistente:

URI:http://hdl.handle.net/10316.2/38318

DOI:

DOI:http://dx.doi.org/10.14195/0871-1623_34_8

Accessed : $\quad$ 26-Apr-2023 15:19:07

A navegação consulta e descarregamento dos títulos inseridos nas Bibliotecas Digitais UC Digitalis, UC Pombalina e UC Impactum, pressupõem a aceitação plena e sem reservas dos Termos e Condições de Uso destas Bibliotecas Digitais, disponíveis em https://digitalis.uc.pt/pt-pt/termos.

Conforme exposto nos referidos Termos e Condições de Uso, o descarregamento de títulos de acesso restrito requer uma licença válida de autorização devendo o utilizador aceder ao(s) documento(s) a partir de um endereço de IP da instituição detentora da supramencionada licença.

Ao utilizador é apenas permitido o descarregamento para uso pessoal, pelo que o emprego do(s) título(s) descarregado(s) para outro fim, designadamente comercial, carece de autorização do respetivo autor ou editor da obra.

Na medida em que todas as obras da UC Digitalis se encontram protegidas pelo Código do Direito de Autor e Direitos Conexos e demais legislação aplicável, toda a cópia, parcial ou total, deste documento, nos casos em que é legalmente admitida, deverá conter ou fazer-se acompanhar por este aviso.

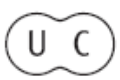




\title{
Fatores de controlo sobre a localização de grandes complexos de ravinas: uma análise com base em reconstrução topográfica e regressão logística Controlling factors of the location of large gully complexes: an analysis based on topographic reconstruction and logistic regression
}

\author{
Rafaello Bergonse \\ Centro de Estudos Geográficos da Universidade de Lisboa \\ rafaellobergonse@campus.ul.pt

\section{Eusébio Reis} \\ Centro de Estudos Geográficos da Universidade de Lisboa \\ eusebioreis@campus.ul.pt
}

\section{Resumo:}

As formações sedimentares terciárias das bacias hidrográficas das ribeiras de Ulme e do Vale do Casal Velho, na margem esquerda da bacia do baixo Tejo, são afetadas por centenas de complexos de ravinas, com dimensões que variam desde pouco menos de $200 \mathrm{~m}^{2}$ até mais de 3 ha de extensão contínua. Estas formas apresentam-se com os fundos assoreados e cobertos de vegetação, evidenciando atividade apenas nas cabeceiras e paredes, na forma de movimentos de massa. No presente trabalho são investigados os fatores responsáveis pela localização destes sistemas de ravinas nas respetivas bacias. Com base na vectorização de 90 destas formas a partir de ortofotografias, investigou-se a influência da topografia pré-erosão, expressa na forma de 8 possíveis variáveis de controlo, com recurso a regressão logística.

Os resultados indicam que a topografia antecedente terá exercido um efeito significativo na determinação dos locais de iniciação destas formas. Esta iniciação terá estado relacionada com um entalhe na rede de drenagem das bacias, originando instabilidade lateral ao longo dos canais. Em locais relativamente declivosos e propensos à acumulação de escoamento sub-superficial, esta instabilidade ter-se-á traduzido no surgimento de ravinas de desnível, percursoras dos grandes complexos de ravinas que hoje evoluem nas vertentes.

Palavras-chave: Ravinamento. Sistemas de ravinas. Complexos de ravinas. Reconstrução topográfica. Regressão logística.

\section{Abstract:}

The Tertiary sedimentary formations of the Ulme and Vale do Casal Velho river basins, on the left margin of the Tagus basin, are affected by hundreds of gully complexes, varying in size from somewhat less than 200 $\mathrm{m}^{2}$ to more than 3 ha. These landforms currently show infilling bottom sectors covered in vegetation. Evidence of present activity, in the form of mass movements, is constrained to the walls and headcuts. In this paper we investigate the factors determining the location of these gully systems in their respective basins. A set of 90 gully complexes were selected and vectorized from aerial ortophotos. Logistic regression was then applied in order to assess the role of 8 potential control variables, obtained through reconstruction of the pre-erosion topography. Results indicate that hillslope topography had a significant effect in determining the places of initiation of the present gully systems. This initiation seems to have been triggered by a downcutting drainage network, originating lateral instability along channel reaches. In places relatively steep and prone to the accumulation of sub-surface drainage, this instability will have caused the initiation of bank gullies, precursor to the large gully complexes that presently evolve on the hillslopes.

Keywords: Gully erosion. Gully systems. Gully complexes. Topographic reconstruction. Logistic regression.

Introdução

As formas resultantes da ação erosiva do escoamento superficial concentrado apresentam uma enorme variabilidade, não só em termos de dimensões e configuração mas também de processos evolutivos
(BERGONSE e REIS, 2011). A partir do seu surgimento, as ravinas frequentemente sofrem bifurcações sucessivas, dando origem a sistemas de canais. Estes podem adquirir uma configuração em rede (canais separados por porções da superfície topográfica não entalhada) (DABA et al., 2003), em complexo (canais 
separados por interflúvios resultantes da degradação da superfície topográfica original) (BUCOLLINI et al., 2012), ou apresentar sectores associados a cada uma destas tipologias (IMESON e KWAAD, 1980).

Os muitos estudos que têm sido dedicados à erosão hídrica concentrada tendem a privilegiar formas relativamente incipientes e ativas, normalmente em contextos agrícolas (AUZET et al., 1995; BELYAEV et al., 2006). Uma vez desenvolvidos, porém, estes entalhes podem permanecer por longos períodos na paisagem, atravessando fases sucessivas de crescimento, assoreamento ou estabilidade. Parkner et al. (2006) estudaram a evolução de numerosas ravinas na Nova Zelândia ao longo do período 1939 2003, definindo fases de expansão e inatividade abrangendo intervalos de vários anos; para uma única ravina na Bélgica, Vanwalleghem et al. (2005) definiram quatro ciclos de entalhe e enchimento estendendo-se ao longo de menos de cem anos.

As abordagens ao estudo de grandes ravinas e sistemas de ravinas têm consistido no estabelecimento de cronologias do seu desenvolvimento baseadas em diferentes métodos de datação (DOTTERWEICH et al., 2003; LANG e MAUZ, 2006), e quantificações da sua evolução ao longo de períodos multi-decenais utilizando fotogrametria (BETTS et al., 2003; PARKNER et al., 2006), por vezes procurando adicionalmente explicar as mudanças registadas com recurso a diferentes fatores (MARTÍNEZ-CASASNOVAS et al., 2004; 2009).

Ao contrário do que acontece com formas menores, a procura de relações entre a presença e as características destes sistemas e a forma da superfície que os antecedeu é dificultada pela sua dimensão, com expressão na informação topográfica de escalas mais detalhadas (1:25000 e maiores). Duas opções se apresentam nestes casos: adotar uma perspetiva regional, caracterizando o enquadramento topográfico destas formas em termos de grandes unidades gerais por oposição à vertente individual (FLÜGEL et al., 2003; BACELLAR et al., 2005); e reconstruir por meio de interpolação a superfície topográfica original. Neste último caso, as abordagens publicadas têm assentado em métodos lineares de interpolação sem recurso a qualquer forma de validação (ALEXANDER et al., 2008; PERROY et al., 2010; BUCCOLINI et al., 2012), ou mesmo em métodos não especificados pelos autores (WELLS e GUTIERREZ, 1982; DABA et al., 2003), colocando em causa a credibilidade dos resultados obtidos.

0 presente trabalho tem por objetivo investigar os controlos sobre a localização dos inúmeros complexos de ravinas que afetam as vertentes das bacias das ribeiras de Ulme e do Vale do Casal Velho, situadas na margem esquerda da bacia do Tejo e tendo as desembocaduras respetivamente a $\mathrm{S}$ e a NE da Chamusca. Um conjunto de 90 destas formas foi selecionado de modo a abranger a gama de variação existente em termos de dimensões, sendo os seus limites vetorizados com base em fotografias aéreas. A reconstituição da topografia que as antecedeu foi depois levada a cabo usando o método de interpolação e a respetiva parametrização que, de entre um total de 24 métodos/parametrizações comparados, apresentou maior capacidade de reproduzir porções da superfície topográfica atual, conforme a metodologia recentemente publicada por Bergonse e Reis (2015). Esta superfície estimada, juntamente com a topografia erodida atual, permitiu obter um conjunto de 8 possíveis variáveis de controlo, expressas na forma de temas cartográficos integrados num Sistema de Informação Geográfica. Recorreu-se finalmente a Regressão Logística para aferir a capacidade destas variáveis para diferenciar locais entalhados de não entalhados, centrando as análises primeiramente na totalidade das áreas afetadas pelos complexos de ravinas e, numa segunda fase, apenas nos seus sectores de desembocadura.

\section{Área de Estudo}

As bacias das ribeiras de Ulme (RU) e do Vale do Casal Velho (RVCV) situam-se na margem esquerda da bacia portuguesa do Tejo, ocupando respetivamente áreas de 138,4 e $12,9 \mathrm{~km}^{2}$, sendo abrangidas na sua quase totalidade pelo município da Chamusca. Ambas as bacias apresentam uma configuração relativamente alongada, estendendo-se nos seus comprimentos máximos por cerca de $23,9 \mathrm{~km}$ no sentido W-E (RU) e por cerca de 5,7 km no sentido NW-SE (RVCV). As altitudes variam entre mínimos de 7 e 20 m (RU e RVCV respetivamente), e máximos de 200 e $198 \mathrm{~m}$, atingidos nos sectores de cabeceira. As precipitações anuais situam-se entre os 600 e os $800 \mathrm{~mm}$ (BRITO, 2005: 51).

Apresentam-se na Figura 1 as unidades morfológicas essenciais das duas bacias e o seu contexto litológico. Este último é dominado por formações clásticas terciárias, cuja distribuição se relaciona fortemente com a morfologia do terreno. As vertentes, com declives médios de $12^{\circ}$ (RU) e $11,4^{\circ}$ (RVCV) são dominadas por uma sucessão de areias, argilas e arenitos (designada Formação Argilo-arenítica ou Complexo Argilo-Gresoso; GONÇALVES et al., 1979; ZBYSZEWSKI e VEIGA FERREIRA, 1979), intercalados com níveis de cascalhos e por vezes de conglomerados, e correspondendo ao Miocénico-Pliocénico. Em contraste, os topos, de carácter aplanado (declives de $2,8^{\circ}$ na RU e de $3,6^{\circ}$ na RVCV), correspondem a extensos mantos de conglomerados e arenitos pliocénicos que definem o nível culminante da bacia terciária do Tejo-Sado (GONÇALVES et al., 1979; ZBYSZEWSKI e VEIGA FERREIRA, 1979; MARTINS, 2004). Para além destas duas formações, largamente dominantes ( $91 \%$ da área da RU e $87,5 \%$ da área da RVCV), o contexto litológico inclui quatro níveis de terraços fluviais $(2,5 \%$ da área da bacia da RU e 6,2\% da área da bacia da RVCV), frequentemente com expressão nas vertentes na forma de rechãs de declive relativamente suave, e depósitos aluvionares ao longo dos fundos dos vales $(6,5 \%$ da área da bacia da RU e 3,3\% da área da bacia da RVCV). As restantes formações ("Areias Superficiais de Vales e de Terraços" e "Granitos Alcalinos de Duas Micas") têm expressão meramente residual. 


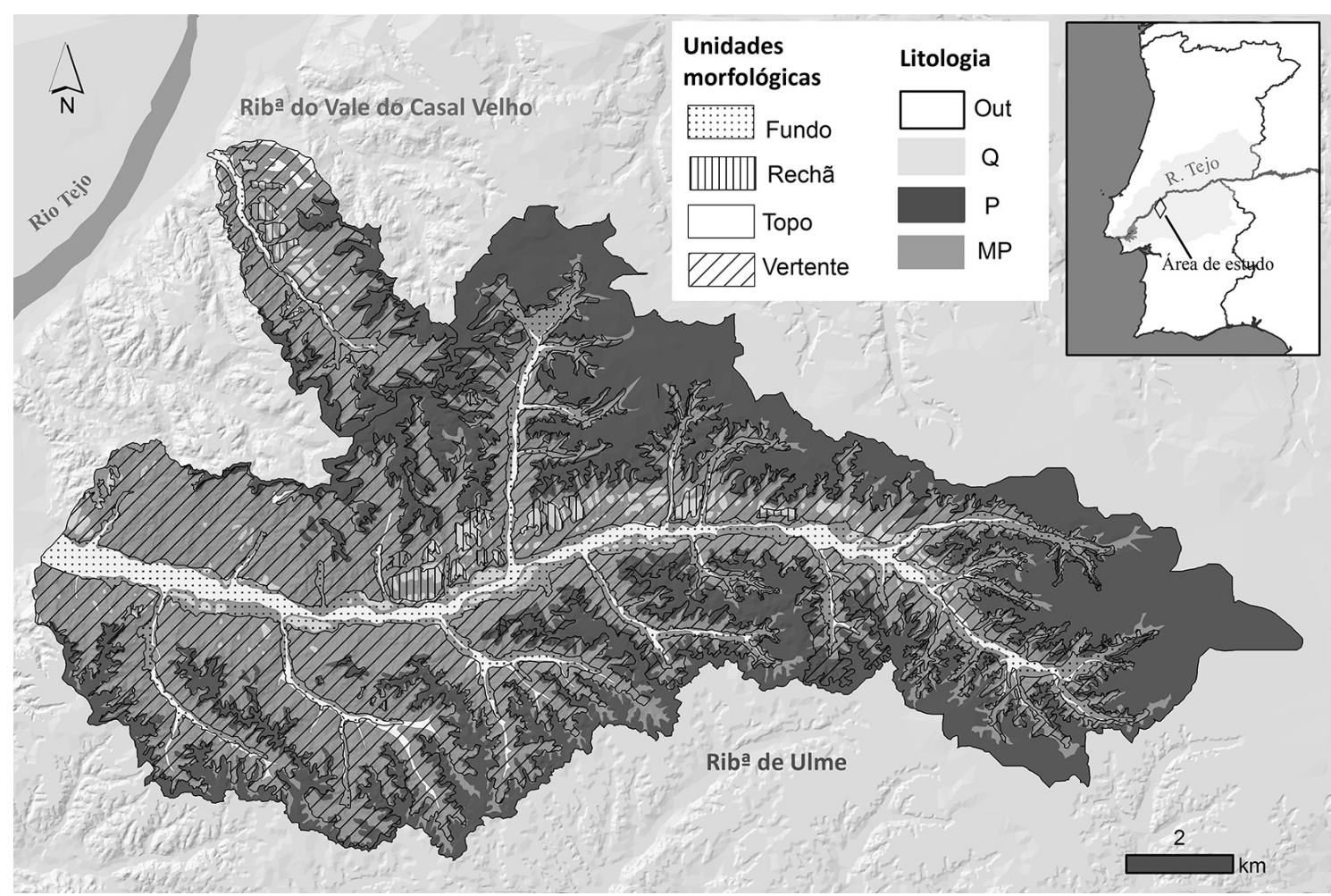

Figura 1

Unidades morfológicas e contexto litológico das duas bacias estudadas, bem como o seu enquadramento face à bacia portuguesa do Tejo (em cima, à direita). Formações litológicas: $(\mathrm{Q})$ - depósitos de terraços fluviais (Plistocénico); $(\mathrm{P})$ - arenitos e conglomerados (Pliocénico); (MP) - formação argilo-arenítica (Miocénico-Pliocénico); (Out) - Outras formações (Aluviões; Areias Superficiais de vales e de terraços; Granitos alcalinos de Duas Micas, porfiróides), agregadas em ordem a facilitar a leitura do mapa. Optou-se por individualizar os Depósitos de Terraços Fluviais em detrimento das Aluviões (que os excedem em área) pelo facto de os complexos de ravinas em estudo ocorrerem sobre as vertentes, e nunca nas formações aluvionares dos fundos de vale.

Fonte: base topográfica, Carta Militar 1:25000; Litologias, Carta Geológica de Portugal à escala 1:50000, Folhas 27-D e 31-B.
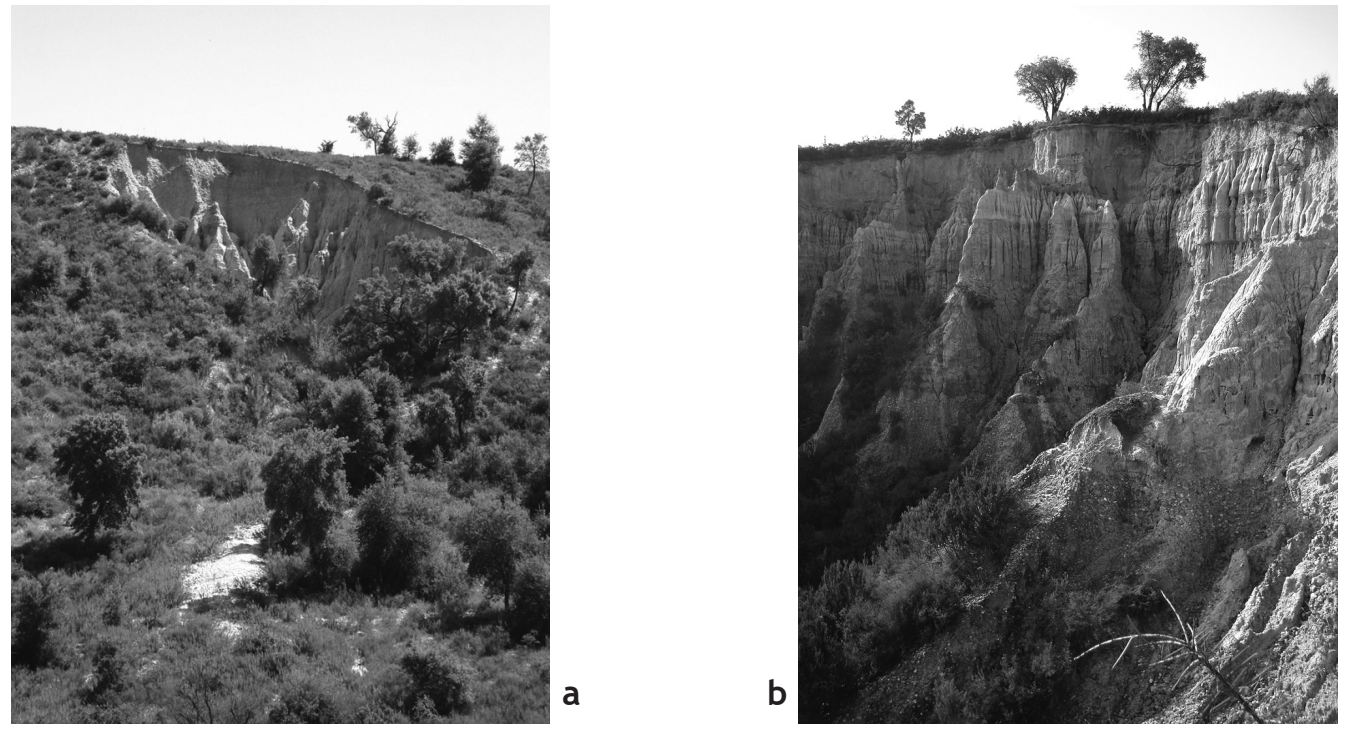

Figura 2

Vista sobre um dos sistemas de ravinas estudados (área $1351 \mathrm{~m}^{2}$, ribeira de Ulme) (a); vista sobre o sector de cabeceira de um sistema de maiores dimensões (área $4881 \mathrm{~m}^{2}$, ribeira do Vale do Casal Velho) (b). Notar paredes sub-verticais desprovidas de vegetação e sectores basais vegetados, apresentando depósitos com origem em movimentos de massa.

Fotografias: R. Bergonse. 
A ocupação do solo é marcada em ambas as bacias pelo eucaliptal, dominando os planaltos cas calhentos e parte das vertentes, e por formações arbustivas espontâneas por entre numerosos exemplares dispersos de Quercus Suber, resultado aparente do abandono e degradação de anterior montado de sobro.

Os solos consistem, de acordo com a classificação dos solos de Portugal desenvolvida pelo SROA (CARVALHO CARDOSO, 1974), em solos Argiluviados Pouco Insaturados, Litólicos e Podzolizados (Carta Complementar de Solos de Portugal à escala 1:25.000).

Ambas as bacias são afetadas por centenas de sistemas de ravinas de grandes dimensões, organizando-se sobretudo em complexo e ocorrendo nas vertentes. As suas dimensões variam entre cerca de $200 \mathrm{~m}^{2}$ e mais de 3 ha de extensão contínua. Encontram-se parcialmente estabilizados, com os fundos assoreados e dominados pela vegetação (Figura 2). $A$ atividade atual é visível apenas nas paredes e cabeceiras, onde extensões sub-verticais e sem vegetação, depósitos basais e árvores com os sistemas de raízes expostos indiciam uma dinâmica baseada em movimentos de massa.

\section{Metodologia}

Selecionou-se um conjunto de 90 sistemas de ravinas a partir da interpretação de ortofotografias ${ }^{1}$ (Figura 3), procurando-se abranger a variabilidade existente em termos de dimensões. Estes sistemas (75,6\% dos quais validados no terreno) foram depois vetorizados na forma de polígonos usando o ArcGIS 10 (software adotado em toda a metodologia descrita).

Apresenta-se no Quadro I uma síntese dos valores apresentados pelas 90 formas quanto à área e ao perímetro, calculados levando em conta os declives das vertentes em que as formas se inserem (BERGONSE e REIS, 2013).

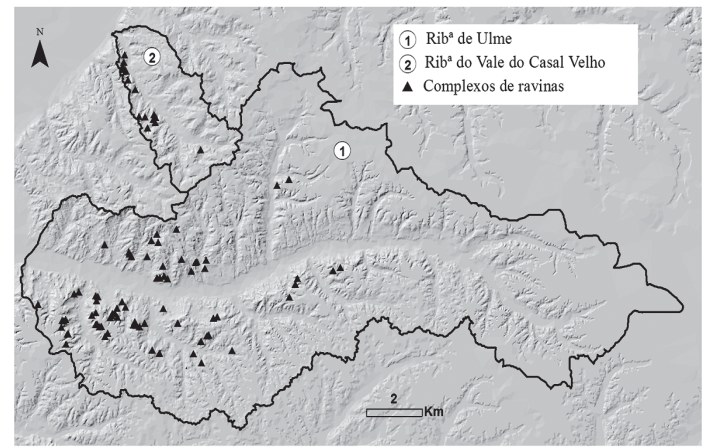

Figura 3

Localização dos 90 complexos de ravinas estudados nas duas bacias. Fonte: Base topográfica, Carta Militar de Portugal 1:25.000.

As ortofotografias, com resolução de 0,5 m, foram produzidas em 2004 no contexto de uma parceria Instituto Geográfico Português/Direcção-Geral dos Recursos Florestais.
Quadro I

Síntese dos valores de área ( $\mathrm{Ar}$ ) e perímetro (Pr) entre os 90 complexos de ravinas estudados.

\begin{tabular}{c|c|c|c|c|c}
\hline & Média & Mín. & Máx. & DP & Total \\
\hline $\operatorname{Ar}(\mathrm{m} 2)$ & 2892,2 & 196,0 & 33151,5 & 4972,9 & 242296,4 \\
\hline $\operatorname{Pr}(\mathrm{m})$ & 206,0 & 56,4 & 1150,3 & 154,3 & 18541,3 \\
\hline
\end{tabular}

Com o fim de procurar compreender a posição dos 90 complexos de ravinas selecionados face às respetivas bacias, adotou-se um conjunto de 8 potenciais variáveis de controlo, identificadas e justificadas no Quadro II.

A dimensão das formas estudadas, fazendo com que tenham expressão na cartografia topográfica adotada $\left(1: 10.000^{2}\right)$, tornou necessário reconstruir a superfície topográfica que as antecedeu. Com este fim em vista, foi desenvolvida uma metodologia para selecionar um método de interpolação óptimo de entre qualquer número de possíveis métodos. Esta assenta na comparação da capacidade dos diferentes métodos para reproduzir áreas amostrais não entalhadas (i.e. de topografia conhecida), sendo descrita em pormenor em Bergonse e Reis (2015). Foram assim comparados 24 métodos de interpolação/parametrizações, sendo definido o algoritmo Topo to Raster, correndo sobre curvas de nível e com a parametrização estabelecida por defeito no ArcGIS 10 , como o método ótimo. Este foi subsequentemente utilizado na interpolação das áreas atualmente entalhadas. O Modelo Numérico de Elevação (MNE) assim produzido, com uma célula de $2 \mathrm{~m}$ de lado, serviu de base à obtenção das variáveis referentes à topografia que antecedeu os sistemas de ravinas, descritas no Quadro II. São exceções a distância à desembocadura das bacias (D_DES) e a área de drenagem (A_DREN), obtidas com base em MNE da topografia atual.

Um exemplo da reconstrução topográfica é apresentado na Figura 4

\section{Aplicação da regressão logística}

A regressão logística pertence à classe mais ampla de técnicas denominadas regressão categorial, adotando o nome de logística quando a variável dependente é nominal dicotómica (MAROCO, 2007: 683). Este é o presente caso, sendo a variável dependente considerada a presença ou ausência de complexos de ravinas.

A função logística permite estimar a probabilidade de ocorrência de uma instância de referência (neste caso 1 por oposição a 0) da variável dicotómica y em estudo, como resultado dos valores de $p$ variáveis independentes, podendo estas ser qualitativas ou quantitativas:

$$
P(y=1)=\frac{\exp \left(\beta_{0}+\beta_{1} x_{1}+\ldots+\beta_{p} x_{p}\right)}{1+\exp \left(\beta_{0}+\beta_{1} x_{1}+\ldots+\beta_{p} x_{p}\right)}
$$

\footnotetext{
A cartografia utilizada foi produzida em 2007 pela empresa Municípia S.A. para a Comunidade Intermunicipal da Lezíria do Tejo, e gentilmente cedida com autorização desta entidade.
} 
Quadro II

As potenciais variáveis de controlo adotadas e a respetiva justificação face ao objetivo do trabalho.

\begin{tabular}{|c|c|}
\hline Variável & Justificação \\
\hline Declive $\left(\mathrm{DEC},{ }^{\circ}\right)$ & Valores elevados promoverão a aceleração do escoamento e a redução da infiltração. \\
\hline $\begin{array}{l}\text { Curvatura de Perfil } \\
\text { (C_PER, adimensional) }\end{array}$ & $\begin{array}{l}\text { Valores positivos: concavidade, promovendo a desaceleração do escoamento e a infiltração. } \\
\text { Valores negativos: convexidade, com o efeito oposto (OLAYA, 2009). }\end{array}$ \\
\hline $\begin{array}{l}\text { Curvatura Transversal } \\
\text { (C_TRA, adimensional) }\end{array}$ & $\begin{array}{l}\text { Valores positivos: convexidade, promovendo dispersão do escoamento (superficial e sub- } \\
\text {-superficial); valores negativos: concavidade, com o efeito oposto (SUMMERFIELD, 1991; } \\
\text { OLAYA, 2009) }\end{array}$ \\
\hline $\begin{array}{l}\text { Índice de Potência do Escoamento } \\
\text { (IPE, adimensional) }\end{array}$ & $\begin{array}{l}\text { Formalizado como A.Tan }(B) \text {, em que A é a área de drenagem específica }\left(\mathrm{m}^{2} / \mathrm{m}\right) \text { e } B \text { o decli- } \\
\text { ve em graus. Valores elevados indicam tendência para a produção de escoamento superfi- } \\
\text { cial erosivo. }\end{array}$ \\
\hline $\begin{array}{l}\text { Índice Topográfico de Encharcamento } \\
\text { (ITE, adimensional) }\end{array}$ & $\begin{array}{l}\text { Formalizado como In (A/Tan }(B)) \text {. Valores elevados indicam tendência para a acumulação do } \\
\text { escoamento na topografia, promovendo o encharcamento dos materiais sub-superficiais. }\end{array}$ \\
\hline $\begin{array}{l}\text { Orientação } \\
\text { (oito classes, referentes aos pontos } \\
\text { cardeais e colaterais) }\end{array}$ & $\begin{array}{l}\text { Potencial controlo sobre a erosão, exercido através da hidrologia das vertentes, vegetação } \\
\text { e comportamento mecânico do rególito (KIRBY, 2004: 35). }\end{array}$ \\
\hline $\begin{array}{l}\text { Distância à desembocadura da bacia } \\
\text { (D_DES, m) }\end{array}$ & $\begin{array}{l}\text { Adotada para investigar possíveis relações entre a posição dos sistemas de ravinas e varia- } \\
\text { ções no nível de base das bacias. }\end{array}$ \\
\hline $\begin{array}{l}\text { Área de drenagem } \\
\left(\text { A_DREN, } \mathrm{m}^{2}\right)\end{array}$ & $\begin{array}{l}\text { Medida na desembocadura de cada sistema de ravinas. Indicador do escoamento potencial- } \\
\text { mente disponível para produzir acção erosiva. }\end{array}$ \\
\hline
\end{tabular}
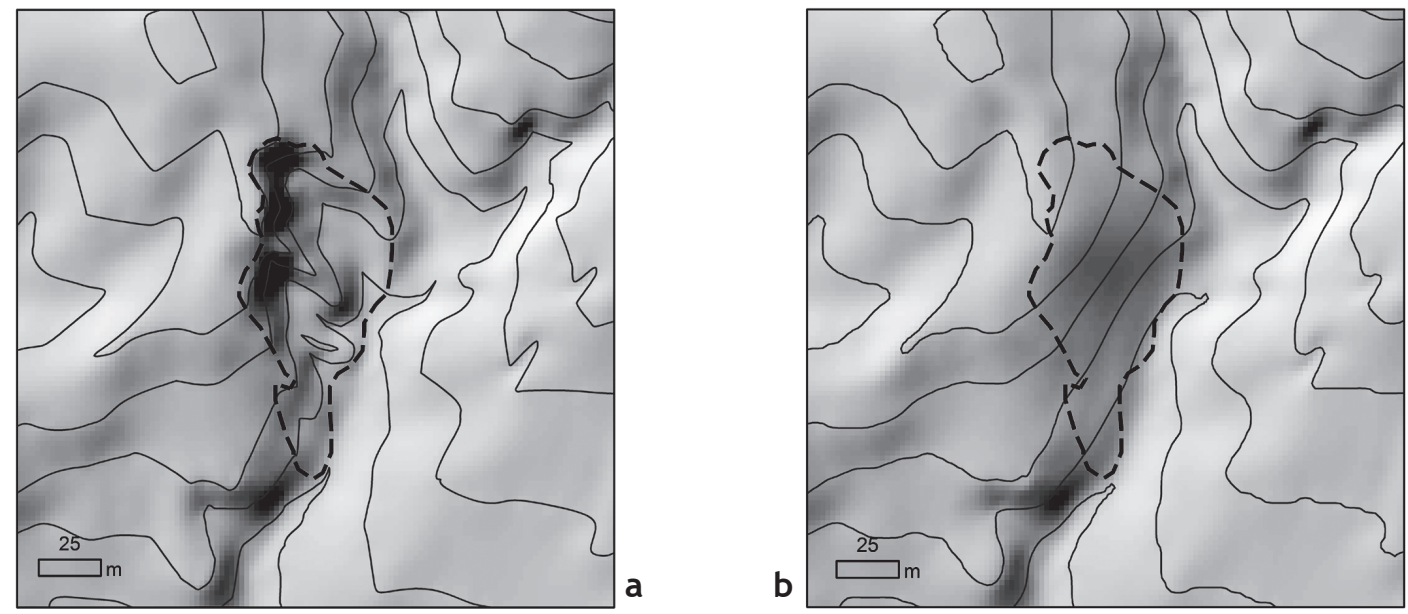

Figura 4

Um exemplo da reconstrução topográfica realizada. (a) - complexo de ravinas no seu estado atual (curvas de nível equidistantes de 5 m); (b) - superfície antecedente reconstruída.

Esta função, cujos resultados são valores entre 0 e 1 , é linearizada com recurso à denominada transformação Logit, adotando a forma:

$$
\ln \left(\frac{P(y=1)}{1-P(y=1)}\right)=\beta_{0}+\beta_{1} x_{1}+\ldots+\beta_{p} x_{p}
$$

É de notar que o resultado desta função (também denominado Logit) é o logaritmo natural da razão entre a probabilidade de ocorrência da instância considerada (1) por oposição à sua não ocorrência (0), ou seja, o logaritmo natural das suas chances (odds) de se verificar. Esta razão é também designada de rácio de verosimilhança (odds ratio). Correspondentemente, os coeficientes de regressão $B_{i}$ não descrevem a variação da variável dependente por unidade de variação de cada independente (tal como ocorre na regressão linear), mas sim a variação no logaritmo das chances de ocorrência da instância considerada quando cada variável independente varia uma unidade. Como os valores de $B_{i}$ são difíceis de interpretar, é habitual interpretar o exponencial destes valores $\operatorname{Exp}\left(B_{i}\right)$, que representa a razão entre os rácios das chances quando $X_{i}$ aumenta uma unidade, e quando $X_{i}$ se mantém igual (MAROCO, 2007:692).

Em função da sua adequação a variáveis categóricas, a regressão logística difere da regressão linear quanto ao método de estimação dos coeficientes de regressão. Por oposição à minimização dos quadrados das diferenças entre valores previstos e observados, é adotado o método da máxima verosimilhança, maximizando a probabilidade de obter as instâncias da variável dependente observadas nas 
unidades de análise a partir das quais se constrói o modelo.

No que diz respeito aos pressupostos, esta técnica não assume normalidade das variáveis independentes, exigindo apenas que não sejam multicolineares (à semelhança do modelo de regressão linear) (MARTÍNEZ-CASASNOVAS et al., 2004: 312).

A regressão logística tem sido frequentemente aplicada ao estudo dos processos erosivos. Meyer e Martínez-Casasnovas (1999) utilizaram-na para prever a localização de ravinas efémeras em vinhas do NE de Espanha com recurso a variáveis topográficas (declive e perfil transversal das vertentes), classificando corretamente $84,6 \%$ das áreas de teste usadas para validação. Mais tarde, Martínez-Casasnovas et al. (2004) recorreram à mesma técnica para explicar a evolução lateral de grandes ravinas na mesma área de estudo. Usando como referência a evolução verificada ao longo de dois períodos de 20 e de 7 anos, classificaram corretamente $87 \%$ das áreas de validação utilizadas, embora não tenham conseguido prever de forma satisfatória os locais onde a expansão das formas efetivamente se verificou. Em Portugal, Martins (2013) aplicou a regressão logística à modelação da suscetibilidade ao ravinamento na área de Pensalvos (vertente $\mathrm{N}$ da serra do Alvão), definindo o declive e, em menor grau, o uso e ocupação do solo como os fatores mais relevantes de predisposição para o surgimento de entalhes.

A análise realizada no presente trabalho estruturou-se em duas etapas sucessivas. Na primeira, averiguou-se a capacidade das variáveis adotadas para diferenciar a topografia antecedente dos sistemas de ravinas (i.e. a topografia reconstruída) face à topografia envolvente, não entalhada. Numa segunda etapa, investigou-se a capacidade das variáveis para diferenciar a topografia que antecedeu especificamente o sector de desembocadura dos sistemas de ravinas face à topografia envolvente. Esta segunda abordagem implicou o pressuposto de que as formas se originaram nestes locais, evoluindo depois através de alargamento e regressão dos sectores de cabeceira (VANDEKERCKHOVE et al., 2000).

Ambas as etapas serão descritas separadamente.

\section{Regressão centrada na totalidade das formas}

Foram geradas de forma aleatória no interior das duas bacias em estudo duas amostras de 400 pontos, sendo uma definida dentro dos 90 polígonos correspondentes aos complexos de ravinas, e outra no exterior. Assegurou-se que todos os polígonos contiveram pontos no interior, sendo estes retirados manualmente das formas com maior concentração quando necessário. No caso dos pontos gerados no exterior dos polígonos, foram movidos manualmente aqueles situados em fundos aplanados de vale e áreas de planalto (onde não há ocorrência de complexos de ravinas), bem como sobre áreas entalhadas.

Os 800 pontos foram depois sobrepostos aos temas cartográficos das possíveis variáveis de controlo (Quadro II), sendo construída uma tabela com os valores de cada variável associados a cada ponto. A única exceção foi a área de drenagem (A_DREN), que se considerou não ter nesta primeira análise relevância. Uma vez que as formas estudadas se estendem frequentemente até ao alto das vertentes, onde as áreas de drenagem são muito reduzidas, os eventuais valores mais elevados obtidos a jusante seriam anulados pelos valores reduzidos a montante. Por conseguinte, esta variável foi utilizada apenas na análise centrada nos sectores de desembocadura, descrita a seguir.

A tabela resultante da sobreposição dos pontos às sete variáveis serviu de base às análises subsequentes, sendo utilizado o software SPSS 16.

Em primeiro lugar, procurou-se definir em que medida cada uma das variáveis permite diferenciar pontos definidos sobre áreas entalhadas e não entalhadas. Desde que seja coerente com o enquadramento teórico do fenómeno em estudo, a existência de diferenças estatisticamente significativas entre as médias das duas distribuições é um importante indicador de que a variável pode ter exercido um efeito relevante sobre a presença das formas, justificando a sua integração no modelo de regressão logística (e.g. MEYER e MARTíNEZ-CASASNOVAS, 1999).

Em ordem a definir o tipo de teste de diferenciação estatística a aplicar (paramétrico ou não paramétrico) foi primeiro necessário verificar, para cada variável independente quantitativa, os dois pressupostos associados aos testes paramétricos. Foram aplicados o teste de Kolmogorov-Smirnov à normalidade e o teste de Levene à homogeneidade das variâncias. Os resultados mostraram que a hipótese de normalidade pode ser rejeitada com uma margem de erro inferior a $1 \%$ para quase todas as variáveis, com DEC a constituir a única exceção.

No caso do teste de Levene, os resultados permitiram rejeitar a hipótese de homogeneidade para a D_DES e a C_PER.

Em função dos valores obtidos, todas as variáveis consideradas não normais foram comparadas usando o teste não paramétrico de Kolmogorov-Smirnov, sendo DEC testado com recurso ao teste t-Student. Os resultados são apresentados no Quadro III e mostram que ITE e DEC não apresentam valores médios significativamente diferentes em áreas entalhadas e não entalhadas.

Quadro III

Resultados dos testes de diferenciação estatística usados para aferir diferenças entre os valores amostrados para áreas não entalhadas e para a topografia pré-erosão.

\begin{tabular}{c|c|c}
\hline Variável & Estatística & a \\
\hline DEC & 1647 & 0,100 \\
\hline D_DES & 6364 & 0,000 \\
\hline ITE & 1273 & 0,078 \\
\hline IPE & 1838 & 0,002 \\
\hline C_TRA & 1874 & 0,002 \\
\hline C_PER & 2546 & 0,000 \\
\hline
\end{tabular}

Nota: À exceção de DEC (a itálico), para o qual se adotou o teste t-Student, todas as variáveis foram testadas com recurso ao teste não-paramétrico de Kolmogorov-Smirnov. Valores significativos estão a negrito. 
Dada a sua natureza categórica, a orientação das vertentes foi comparada usando o teste do Qui-quadrado $\left(\mathrm{x}^{2}\right)$ (MAROCO, 2007:103). Os resultados mostraram que a hipótese $\left(\mathrm{H}_{0}\right)$ de igualdade entre as frequências de cada classe de orientação para locais entalhados e não entalhados pode ser rejeitada com uma margem de erro inferior a 0,001. Esta diferença muito significativa é claramente visível num gráfico de barras representando as percentagens de pontos associados às diferentes orientações em áreas entalhadas e não entalhadas (Figura 5).

Ao mesmo tempo, a Figura mostra claramente que os pontos em áreas entalhadas apresentam sobretudo orientações a $\mathrm{E}, \mathrm{SE}$ e $\mathrm{W}$, padrão que não corresponde ao contraste $\mathrm{N}-\mathrm{S}$ através do qual a orientação das vertentes exerce o seu efeito máximo. A análise subsequente do padrão de distribuição dos sistemas de ravinas face à topografia mostrou que as orientações dominantes refletem apenas o traçado dos vales em cujas vertentes as formas ocorrem, pelo que esta variável foi considerada irrelevante como controlo sobre a localização.

Uma vez definidas as variáveis a integrar na análise (D_DES, IPE, C_TRA, C_PER), analisaram-se os coeficientes de correlação linear de Pearson $(R)$ a fim de detetar eventuais situações de multicolinearidade. Obteve-se um $R$ máximo de 0,331 (valor absoluto) permitindo assumir independência entre as variáveis.

Foi por fim construído o modelo de regressão logística ${ }^{3}$. Do conjunto de 800 pontos, foi extraído um sub-conjunto de 267 (um terço do total). Este sub-conjunto foi guardado para validação independente do modelo, para cuja construção foram usados os restantes 533 pontos. Após a obtenção dos resultados, foram analisados os resultados do teste de Wald, calculados automaticamente pelo SPSS e destinados a testar a hipótese nula de que cada um dos coeficientes de regressão é igual a 0 , ou seja, não tem qualquer contributo para o modelo (MAROCO, 2007: 700). De todas as variáveis, apenas D_DES se mostrou significativa para uma margem de erro de
$5 \%$ (obteve $a<0,001$ ), sendo todas as restantes eliminadas (IPE com $\alpha=0,564$; C_TRA com 0,901 e C_PER com 0,080). Foi novamente corrido o processo de modelação com a única variável significativa, sendo os resultados apresentados no Quadro IV juntamente com os valores associados aos testes de significância e qualidade do modelo, a seguir descritos.

\section{Quadro IV}

Resultados da regressão utilizando a única variável independente considerada significativa segundo o teste de Wald. B - Coeficientes de regressão logística; Wald - Estatística de Wald; a - margem de erro associada à rejeição da $\mathrm{H}_{0}$ (i.e. $\mathrm{B}=0$ ).

\begin{tabular}{|c|c|c|c|c|}
\hline & B & $\operatorname{Exp}(B)$ & Wald & a \\
\hline Intercepto & 2,681410838 & 14,60568502 & 98,61 & 0,000 \\
\hline D_des & $-0,000200527$ & 0,999799493 & 103,57 & 0,000 \\
\hline \multicolumn{5}{|c|}{ Teste do Rácio de Verosimilhança: $a=0,000$} \\
\hline \multicolumn{5}{|c|}{ Teste de ajustamento de Hosmer e Lemeshow: $a=0,000$} \\
\hline \multicolumn{5}{|c|}{ pseudo- $R^{2}$ Nagelkerke: 0,322} \\
\hline
\end{tabular}

O teste do Rácio de Verosimilhanças pretende testar a significância do modelo ajustado com base na razão entre a verosimilhança de um modelo com todas as variáveis face à de outro só com o intercepto. No presente caso, o valor de a é extremamente baixo $(0,000)$, mostrando que o modelo é estatisticamente muito significativo ou, por outras palavras, que a variável independente influencia o logit (MAROCO, 2007: 697), em concordância com a significação obtida no teste de Wald. Por oposição, o resultado do teste de ajustamento de Hosmer e Lemeshow permite rejeitar a $\mathrm{H}_{0}$, com uma margem de erro extremamente baixa, indicando um fraco ajustamento entre o modelo e os dados que este pretende reproduzir (MAROCO, 2007: 699). Finalmente, o pseudo- $R^{2}$ de Nagelkerke, variando entre 0 e 1 e calculado pelo SPSS como uma aproximação à proporção da variância da variável dependente explicada pelo modelo (MARTÍNEZ-CASASNOVAS et al., 2004: 315; MAROCO, 2007: 701) produziu um valor de apenas 0,322 .

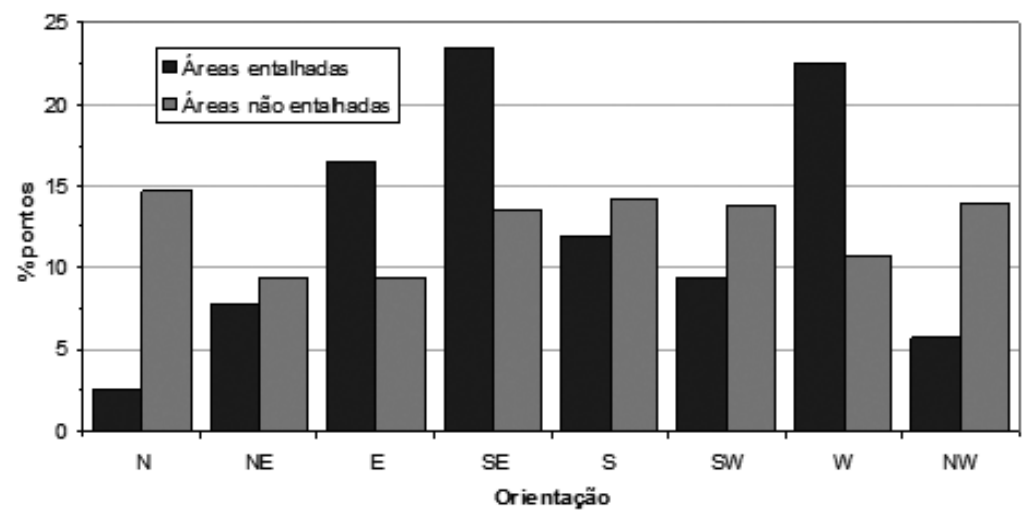

Figura 5

Percentagens dos pontos amostrais associados a diferentes classes de orientação em áreas entalhadas e não entalhadas.

\footnotetext{
3 Utilizou-se o método Enter, definido por defeito no software SPSS 16, e segundo o qual todas as variáveis são inseridas em simultâneo.
} 
De forma a validar independentemente os resultados do modelo e assim quantificar a sua capacidade discriminante, a equação associada foi operacionalizada para os 267 pontos anteriormente guardados para validação com recurso aos coeficientes apresentados no Quadro IV. A formulação do modelo construído é, assim:

$$
p(r v)=\frac{e^{\left(2,681410838-0,000200527 . D_{-} d e s\right)}}{1+e^{\left(2,681410838-0,000200527 . D_{-} d e s\right)}}
$$

em que $p(r v)$ é a probabilidade de cada unidade de análise (i.e. cada ponto amostral) se situar numa área entalhada, variando entre 0 e 1 . A equação foi implementada no Microsoft Excel, sendo definido o limiar de 0,5 para definir uma área como estando ou não afetada por ravinas. Os resultados da validação utilizando os 267 pontos são apresentados no Quadro V.

A análise dos resultados mostra que o modelo prevê razoavelmente a presença e a ausência de complexos de ravinas, tendo classificado corretamente $68,9 \%$ das unidades de análise. Percentagens muito próximas foram obtidas para a sensibilidade e a especificidade, ou seja, a capacidade do modelo para prever, respetivamente, valores positivos na presença do fenómeno, e valores negativos na sua ausência (e.g. ALVAREZ-ARBESÚ e FELICÍSIMO, 2002: 221; MAROCO, 2007: 709).

Foi também calculada a área sob a curva ROC (Receiver Operating Characteristic). Este indicador pretende caracterizar a capacidade de um classificador para discriminar o fenómeno de interesse na sua presença e de minimizar as classificações erradas na sua ausência (i.e. erros de comissão). É obtido criando um gráfico de dispersão em que para todos os possíveis limiares de probabilidade adotados para discriminar presença vs. ausência, são cruzadas a sensibilidade (eixo dos yy) e 1-especificidade, ou seja, a proporção de falsos positivos (eixo dos $x x$ ). A curva ROC tem a vantagem de comparar todos os possíveis limiares de um modelo, por oposição a um limiar arbitrariamente definido (HILL e LEWICKI, 2006: 707), e tem como referências valo- res de 1 (máxima capacidade para identificar o fenómeno na sua presença e de não o identificar erradamente na sua ausência) e 0,5 (modelo aleatório, sem qualquer capacidade de classificação). No presente caso, o valor obtido de 0,76 mostra claramente que o modelo possui algum poder discriminante. De acordo com os valores de referência propostos por Hosmer e Lemeshow (2000, citados em MAROCO, 2007: 710), o modelo revela-se próximo do limite entre uma discriminação "aceitável" e "boa" $(0,80)$.

Quadro V

Resultados da validação do modelo de regressão logística com recurso ao conjunto independente de 267 pontos.

\begin{tabular}{|c|c|c|c|}
\hline \multirow[b]{2}{*}{ Valores observados } & \multicolumn{2}{|c|}{ Valores previstos } & \multirow[b]{2}{*}{ Total } \\
\hline & $P 1(p=>0,5)$ & $\begin{array}{c}P 0 \\
(P<0,5)\end{array}$ & \\
\hline 01 & 91 & 36 & 127 \\
\hline \multirow[t]{2}{*}{$\mathrm{OO}$} & 47 & 93 & 140 \\
\hline & 138 & 129 & 267 \\
\hline \multicolumn{3}{|c|}{ Sensibilidade (P101/Total 01*100) (\%) } & 71,7 \\
\hline \multicolumn{3}{|c|}{ Especificidade (P0O0/total O0*100) (\%) } & 66,4 \\
\hline \multicolumn{3}{|c|}{ Percentagem de valores corretamente previstos } & 68,9 \\
\hline \multicolumn{3}{|l|}{ Área sob a curva ROC } & 0,76 \\
\hline
\end{tabular}

Por fim, foi criado um gráfico de barras representando a distribuição dos valores observados para os 267 pontos (i.e. 0 ou 1) pelas probabilidades previstas (Figura 6). A sua análise confirma a capacidade do modelo para diferenciar razoavelmente as duas classes opostas. Ao mesmo tempo, torna evidente que o modelo identifica melhor áreas entalhadas do que não entalhadas, já que há claramente uma maior concentração dos pontos com valor 1 nas classes de probabilidade elevada do que o oposto. É também de notar que a quantidade de pontos classificados como entalhados não aumenta claramente com o aumento da probabilidade. Este desajustamento é refletido pelo fraco resultado obtido no teste de Hosmer e Lemeshow (Quadro IV).

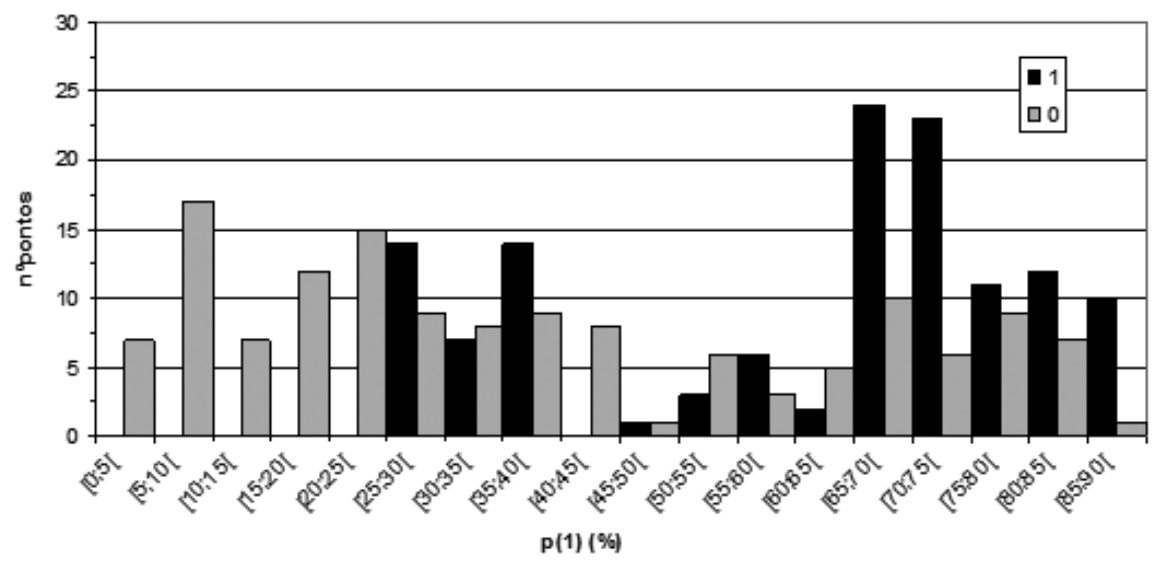

Figura 6

Distribuição dos pontos sobre áreas entalhadas (1) e não entalhadas (0) pelos valores de probabilidade calculados com relação ao conjunto de 267 pontos para validação (expressos em percentagem). 


\section{Regressão centrada nas desembocaduras}

Pretendeu-se com a segunda etapa da metodologia testar a hipótese de que os complexos de ravinas se originaram a partir da atual desembocadura, regredindo depois para montante. De acordo com esta hipótese, a topografia que antecedeu a generalidade da área atualmente ocupada pelas formas (considerada na análise anteriormente descrita) não seria necessariamente diferente da envolvente, tendo o seu entalhe sido ditado pelo recuo das cabeceiras a partir dos locais de iniciação.

Aplicou-se novamente a regressão logística com o objetivo de testar a capacidade das várias possíveis variáveis de controlo (Quadro II) para diferenciar a topografia não erodida envolvente aos complexos de ravinas daquela que antecedeu especificamente os seus sectores de desembocadura. Estes sectores foram vetorizados na forma de linhas para os 90 sistemas de ravinas, com o apoio da análise visual dos polígonos foto-interpretados e de um relevo de sombras. Ao longo destas linhas, gerou-se depois aleatoriamente um conjunto de 400 pontos, separados entre si por uma distância mínima de $2 \mathrm{~m}$ (dimensão da célula do MNE com a topografia reconstruída). Uma análise subsequente do conjunto de pontos permitiu assegurar que todos os 90 sistemas de ravinas tiveram pelo menos um ponto na desembocadura, sendo estes retirados das formas com maior quantidade quando necessário.

Finalmente, e à semelhança dos procedimentos adotados com relação ao primeiro modelo construído, extraíram-se para os 800 pontos amostrais os valores de IPE, ITE, DEC, C_TRA, C_PER, e D_DES, sendo agora considerada também A_DREN. A tabela com todos os valores foi exportada para o SPSS 16, sendo realizada a sequência de análises já descrita na primeira etapa do trabalho (testes de normalidade e de homogeneidade das variâncias, testes de diferenciação estatística, análise de correlação, regressão).

Foi possível rejeitar significativamente a hipótese de normalidade para os conjuntos de valores associados a todas as variáveis e tanto para os conjuntos de pontos nas desembocaduras das formas como na topografia envolvente. A única exceção foi o declive (DEC), em que apenas foi possível rejeitar a normalidade para os pontos nas desembocaduras dos sistemas de ravinas. Neste caso particular, optou-se por avaliar a significação das diferenças entre as médias dos dois conjuntos recorrendo em simultâneo a testes paramétricos (t-Student) e não paramétricos (Kolmogorov-Smirnov). Os resultados foram coincidentes, determinando que os dois conjuntos de pontos apresentam médias significativamente diferentes. 0 mesmo foi determinado em relação às restantes variáveis (Quadro VI).

A matriz de correlação obtida para as sete variáveis permitiu observar um $R$ máximo de 0,525 , considerando-se que, tal como na análise anterior, não se verificaram situações de multicolinearidade entre as variáveis independentes.
Quadro VI

Resultados dos testes de diferenciacão estatística usados para aferir diferenças entre os pontos amostrais em áreas não entalhadas e nos sectores de desembocadura das atuais formas erosivas. À exceção do declive, para o qual se adotou adicionalmente o teste t-Student (valores em itálico), todas as variáveis foram testadas com recurso ao teste não-paramétrico de Kolmogorov-Smirnov.

\begin{tabular}{c|c|c}
\hline \multicolumn{2}{c}{ Variável } & Estatística \\
\hline DEC & 2,369 & 0,000 \\
\hline DEC & 3,397 & 0,001 \\
\hline D_DES & 7,071 & 0,000 \\
\hline ITE & 3,677 & 0,000 \\
\hline IPE & 6,046 & 0,000 \\
\hline C_TRA & 4,384 & 0,000 \\
\hline C_PER & 4,808 & 0,000 \\
\hline A_DREN & 1,909 & 0,001 \\
\hline
\end{tabular}

Após a seleção aleatória de 533 pontos para modelação (i.e. dois terços do total) destinando os restantes 267 para validação, foi corrido o algoritmo de regressão. A análise do resultado permitiu, à semelhança do procedimento anteriormente seguido, eliminar as variáveis cujos coeficientes de regressão não se revelaram significativamente diferentes de 0 $(\alpha \leq 0,05)$ no teste de Wald: IPE $(\alpha=0,147)$, C_TRA $(\alpha=0,869)$ e A_DREN $(\alpha=0,222)$. Foi depois construído o modelo com as quatro variáveis restantes. Os resultados são apresentados no Quadro VII, juntamente com os testes de significância e qualidade.

0 modelo é considerado estatisticamente muito significativo de acordo com o teste do Rácio de Verosimilhanças. Ao contrário da regressão anterior, também o Teste de Ajustamento de Hosmer e Lemeshow permite agora assumir o modelo como significativo, não se podendo (para a margem de erro adotada de 0,05 ) rejeitar a hipótese nula de que este se ajusta aos dados. Por último o pseudo- $\mathrm{R}^{2}$ de $\mathrm{Na}$ gelkerke foi de 0,544 , valor bastante superior aos 0,322 anteriormente obtidos (Quadro IV).

Quadro VII

Resultados da regressão logística utilizando as quatro variáveis consideradas significativas segundo o teste de Wald. B - Coeficientes de regressão logística; Wald - Estatística de Wald; $a$ - margem de erro associada à rejeição da $H_{0}$ (i.e. $B=0$ ).

\begin{tabular}{c|c|c|c|c}
\hline & B & $\operatorname{Exp}(\mathrm{b})$ & Wald & $\mathrm{a}$ \\
\hline Intercepto & $-2,647047105$ & 0,070860147 & 14,40 & 0,000 \\
\hline D_DES & $-0,000225464$ & 0,999774562 & 79,84 & 0,000 \\
\hline ITE & 0,57194414 & 1,771708153 & 39,12 & 0,000 \\
\hline DEC & 0,099550579 & 1,104674343 & 21,36 & 0,000 \\
\hline C_PER & 0,504484988 & 1,656132373 & 35,94 & 0,000 \\
\hline
\end{tabular}

Teste do Rácio de Verosimilhanças: $a=0,000$

Teste de ajustamento de Hosmer e Lemeshow: $\alpha=0,06$ pseudo- $R^{2}$ Nagelkerke: 0,544

Experimentou-se retirar do modelo a distância à desembocadura a fim de aferir a importância específica da topografia das vertentes na iniciação dos sistemas de ravinas. Como consequência, o valor de pseudo- $R^{2}$ desceu para 0,348 , e deixou de se poder considerar o modelo ajustado aos dados segundo o Teste de Hosmer e Lemeshow $(\alpha=0,005)$. 
A equação de regressão foi construída com base nos coeficientes obtidos e depois implementada no Microsoft Excel, sendo utilizada para classificar os 267 pontos de validação. Os resultados são sintetizados no Quadro VIII.

\section{Quadro VIII}

Validação do modelo de regressão logística com recurso ao conjunto independente de 267 pontos.

\begin{tabular}{|c|c|c|c|}
\hline & \multicolumn{2}{|c|}{ Valores previstos } & \multirow[b]{2}{*}{ Total } \\
\hline Valores observados & $P 1(p=>0,5)$ & $P 0(p<0,5)$ & \\
\hline 01 & 106 & 24 & 130 \\
\hline \multirow[t]{2}{*}{$\mathrm{OO}$} & 43 & 94 & 137 \\
\hline & 149 & 118 & 267 \\
\hline \multicolumn{3}{|c|}{ Sensibilidade (P101/Total 01*100) } & 81,5 \\
\hline \multicolumn{3}{|c|}{ Especificidade (P000/Total $00 * 100)$} & 68,6 \\
\hline \multicolumn{3}{|c|}{ Percentagem de valores corretamente previstos } & 74,9 \\
\hline \multicolumn{3}{|c|}{ Área sob a curva ROC } & 0,84 \\
\hline
\end{tabular}

Conforme o Quadro VIII, o modelo permitiu classificar corretamente $81,5 \%$ dos pontos amostrais situados em áreas erodidas e obteve um valor de 0,84 da área sob a curva ROC, correspondendo, segundo Hosmer e Lemeshow (2000; citados em MAROCO, 2007: 710), a uma boa discriminação. A sua expressão gráfica é apresentada na Figura 7, evidenciando a capacidade das variáveis independentes para classificar corretamente pontos em áreas erodidas e não erodidas (em contraste com os resultados obtidos considerando a totalidade das formas, apresentados na Figura 6).

\section{Discussão e conclusões}

Em ordem a facilitar a análise e comparação dos resultados, apresentam-se no Quadro IX os valores médios das variáveis integradas nos dois modelos de regressão logística construídos, para os conjuntos de pontos associados a complexos de ravinas e à topografia envolvente.

Quadro IX

Valores médios das variáveis consideradas significativas nos dois modelos construídos, para os conjuntos de pontos em áreas erodidas (1) e não erodidas (0).

\begin{tabular}{|c|c|c|c|c|c|}
\hline & \multirow{2}{*}{$\begin{array}{l}\text { Modelo } 1 \\
\text { D_DES (m) }\end{array}$} & \multicolumn{4}{|c|}{ Modelo 2} \\
\hline & & $\operatorname{DEC}\left({ }^{\circ}\right)$ & D_DES (m) & ITE & C_PER \\
\hline 0 & 13098 & 16,5 & 15850 & 4,4 & 0,1 \\
\hline 1 & 7212 & 18,2 & 6990 & 5,3 & 1,7 \\
\hline
\end{tabular}

Ambos os modelos de regressão logística construídos demonstraram uma relação significativa entre a localização dos complexos de ravinas e D_DES, evidenciando que as formas se concentram nos sectores jusante das respetivas bacias.

A primeira modelação realizada tornou claro que as propriedades topográficas não permitem, por si, discriminar áreas entalhadas e não entalhadas. Em contraste, o segundo modelo demonstrou que a topografia que antecedeu os sectores de desembocadura dos sistemas de ravinas (assumidos como locais de iniciação) os discrimina significativamente face às áreas não entalhadas. Estes resultados indicam que a morfologia das vertentes terá sido significativa na determinação do local de iniciação dos complexos de ravinas. A partir deste ponto, a evolução destes sistemas terá sido sobretudo controlada por outros fatores.

De acordo com os valores apresentados no Quadro IX, as formas ter-se-ão iniciado em locais relativamente declivosos, de perfil relativamente côncavo (promovendo a desaceleração e infiltração do escoamento por oposição à sua aceleração), e ao mesmo tempo com tendência para a concentração sub-superficial do escoamento (elevado ITE). Juntamente com o facto adicional de o IPE não se ter revelado significativo, estes resultados indicam que

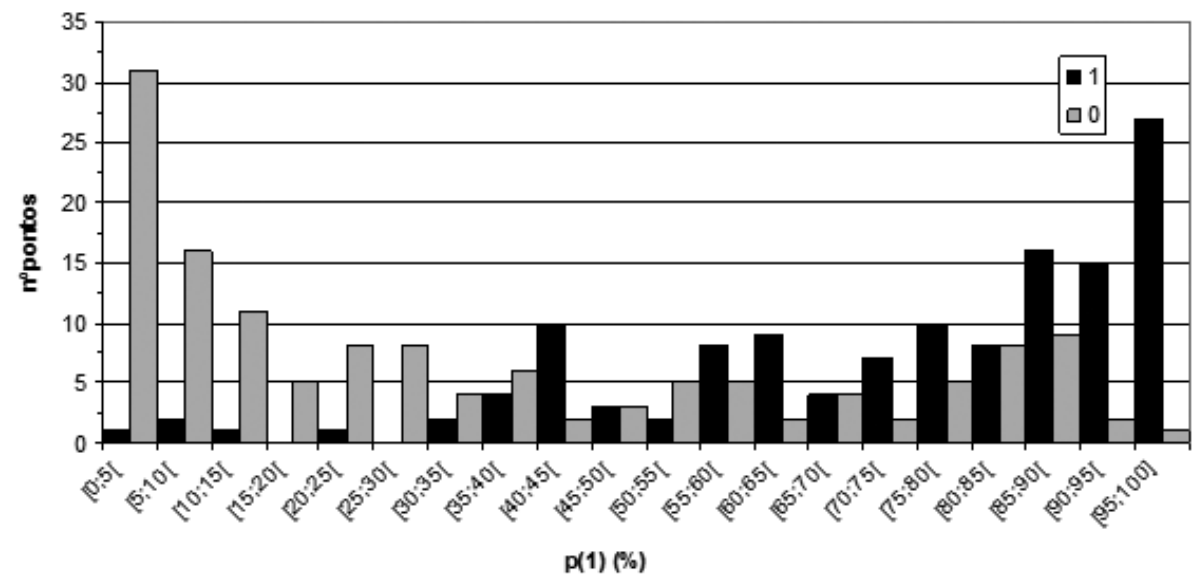

Figura 7

Distribuição dos pontos sobre áreas entalhadas (1) e não entalhadas (0) pelos valores de probabilidade calculados com relação ao conjunto de 267 pontos para validação (expressos em percentagem), usando o modelo centrado nas áreas de desembocadura. 
um teor em água relativamente elevado nos materiais sub-superficiais terá sido um fator preponderante na iniciação dos complexos de ravinas. A relação direta que existe entre o teor em humidade dos materiais e o seu comportamento mecânico sugere aqui um papel importante por parte dos movimentos de massa, por oposição ao entalhe associado ao escoamento superficial.

O facto de (tal como o IPE) a área de drenagem (A_DREN) não se ter relevado significativa na discriminação das desembocaduras sugere que a iniciação das formas não foi determinada pelo escoamento proveniente de montante. A tendência dos complexos de ravinas para se localizaram em relativa proximidade à desembocadura das respetivas bacias vem, assim, indicar que a sua iniciação se relacionou com a dinâmica da rede de drenagem a jusante, da qual são tributários.

Um entalhe da rede de drenagem parece portanto ter sido o fator de iniciação das formas em estudo, promovendo a instabilidade ao longo dos canais. Esta instabilidade teria incidido particularmente sobre locais ao mesmo tempo declivosos e propensos à acumulação do escoamento sub-superficial, motivando a ocorrência de colapsos e a instalação de ravinas de desnível, cuja expansão posterior para montante terá dado lugar aos grandes complexos de ravinas que hoje degradam as vertentes.

Em harmonia com o objetivo inicialmente definido, a metodologia aplicada permitiu explicar em grande parte a localização dos complexos de ravinas que afetam as bacias das ribeiras de Ulme do Vale do Casal Velho. Ao mesmo tempo, os resultados deixam em aberto algumas questões para investigação futura, relacionadas com dois pontos essenciais.

0 primeiro prende-se com a cronologia e as causas do entalhe que terá determinado a iniciação das formas. Duas possibilidades afiguram-se como plausíveis: uma descida do nível de base, e a ocorrência de um ou vários episódios de escoamento excecional. Qualquer uma delas implicaria um efeito erosivo acrescido no sector terminal das bacias.

O segundo prende-se com a dinâmica evolutiva dos complexos de ravinas após a iniciação, e até ao seu estado atual de estabilização parcial. Neste contexto, seria relevante determinar não só a cronologia desta evolução, mas também os controlos sobre a enorme variabilidade morfológica atualmente observável.

\section{Bibliografia}

ALEXANDER, R. W.; CALVO-CASES, A.; ARNAU-ROSALÉN, E.; MATHER, A. E. e LÁZARO-SUAU, R. (2008) - "Erosion and stabilization sequences in relation to base level changes in the El Cautivo badlands, SE Spain". Geomorphology, 100, pp. 83-90.

ALVAREZ-ARBESÚ, R. e FELICÍSIMO, A. (2002) - "GIS and logistic regression as tools for environmental management: a coastal cliff vegetation model in
Northern Spain”. In: BREBBIA, C. A. e PASCOLO, P. (Eds.) - Management Information Systems 2002: GIS and Remote Sensing. Wessex Institute of Technology Press, pp. 215-224.

AUZET, A.; BOIFFIN, J. e LUDWIG, B. (1995) - "Concentrated flow erosion in cultivated catchments: influence of soil surface state". Earth Surface Processes and Landforms, Vol. 20, pp. 759-767.

BACELLAR, L. de A. P.; COELHO NETTO, A. L. e LACERDA, W. A. (2005) - "Controlling factors of gullying in the Maracujá catchment, Southeastern Brazil”. Earth Surface Processes and Landforms, 30, pp. 1369-1385.

BELYAEV, V. R.; SIDORCHUK. A.; GOLOSOV, V. N.; WALLBRINK, P. J. e MURRAY, A. S. (2006) - "Assessing the contribution of different processes to soil degradation within an arable catchment of the Stavropol upland, Southern European Russia". In: OWENS, P. N. e COLLINS, A. J. (Eds.) - Soil Erosion and Sediment Redistribution in River Carchments. CAB International, pp. 40-50.

BERGONSE, R. e REIS, E. (2011) - "Formas, processos e padrões na erosão por ravinamento: para um enquadramento teórico coerente". Finisterra - Revista Portuguesa da Geografia, Vol. XLVI (92), pp. 99-120.

BERGONSE, R. e REIS, E. (2013) - “Medições lineares e areais de formas do relevo em SIG: quantificação do erro inerente à assunção de topografia plana e implicações para a investigação". In:: NUNES, A; CUNHA, L.; SANTOS, J.; RAMOS, A.; FERREIRA, R.; PAIVA, I. e DIMUCCIO, L. (Eds.) - Atas do VI Congresso Nacional de Geomorfologia, Coimbra, 21-23 de Fevereiro, pp. 228-232.

BERGONSE, R. e REIS, E. (2015) - "Reconstructing pre-erosion topography using spatial interpolation techniques: a validation-based approach". Journal of Geographical Sciences, Vol. 25(2), pp. 196-210. DOI 10.1007/s11442 015-1162-2.

BETTS, H. D.; TRUSTRUM, N. A. e DE ROSE, R. C. (2003) "Geomorphic changes in a complex gully system measured from sequential digital elevation models, and implications for management". Earth Surface Processes and Landforms 28, pp. 1043-1058.

BRITO, R. S. (2005) - "Clima e suas influências". In: JULIÃO, R. P. e FERNANDES, J. N. (Coords.) - Atlas de Portugal. Instituto Geográfico Português, pp. 50-65.

BUCCOLINI, M.; COCO, L.; CAPPADONIA, C. e ROTIGLIANO, E. (2012) - "Relationships between a new slope morphometric index and calanchi erosion in northern Sicily, Italy". Geomorphology 149-150, pp. 41-48.

CARVALHO CARDOSO, J. V. J. (1974) - “A classificação de solos de Portugal”. Boletim de Solos do SROA, $\mathrm{n}^{\circ} 17$, 
pp. 14-46. Serviço de Reconhecimento e Ordenamento Agrário, Lisboa.

DABA, S.; RIEGER, W. e STRAUSS, P. (2003) - "Assessment of gully erosion in eastern Ethiopia using photogrammetric techniques". Catena, 50, pp. 273-291.

DOTTERWEICH, M.; SCHMITT, A.; SCHMIDTSCHEN, G. e BORK, H. (2003) - Quantifying historical gully erosion in northern Bavaria. Catena, 50, pp.135-140.

FLÜGEL, W.; MÄRKER, M.; MORETTI, S.; RODOLFI, G. e SIDORCHUK, A. (2003) - "Integrating geographical information systems, remote sensing, ground truthing and modelling approaches for regional classification of semi-arid catchments in South Africa". Hydrological Processes, 17, pp. 929-942.

GONÇALVES, F.; ZBYSZEWSKI, G. e CARVALHOSA, A. (1979) - Carta Geológica de Portugal na escala 1:50000. Notícia explicativa da folha 27-D, Abrantes. Serviços Geológicos de Portugal, Lisboa.

HILL, T. e LEWICKI, P. (2006) - Statistics: Methods and Applications. 1st Edition. Statsoft. 832 p.

HOSMER, D. W. e LEMESHOW, S. (2000) - Applied Logistic Regression. 2nd Edition. John Wiley and Sons.

IMESON, A. C. e KWAAD, F. J. P. M. (1980) - "Gully types and gully prediction". K.N.A.G. Geografisch Tijdschrift Vol.14 (5), pp. 430-441.

KIRKBY, M. J. (2004) - "Aspect and Geomorphology". In: GOUDIE, A. S. (Ed.) - Encyclopedia of Geomorphology. Routledge, pp 34-36.

LANG, A. e MAUZ, B. (2006) - Towards chronologies of gully formation: optical dating of gully fill sediments from Central Europe. Quaternary Science Reviews 25, pp. 2666-2675

MAROCO, J. (2007) - Análise Estatística Com Utilização do SPSS. Edições Sílabo. $3^{\mathrm{a}}$ Edição. $822 \mathrm{p}$.

MARTínEZ-CASASNOVAS, J. A.; RAMOS, M. C.e POESEN, J. (2004) - "Assessment of sidewall erosion in large gullies using multi-temporal DEMs and logistic regression analysis". Geomorphology, 58, pp. 305-321.

MARTÍNEZ-CASASNOVAS, J. A.; RAMOS, M. C. e GARCIAHERNÁNDEZ, D. (2009) - "Effects of land-use changes in vegetation cover and sidewall erosion in a gully head of the Penedès region (northeast Spain)". Earth Surface Processes and Landforms, 34, pp. 1927-1937.

MARTINS, B. (2013) - "Cartografia de susceptibilidade ao ravinamento na área de Pensalvos (vertente norte da serra do Alvão)". In: LOURENÇO, L, e MATEUS, M.
(Coords.) - Riscos Naturais, Antrópicos e Mistos. Homenagem ao Professor Doutor Fernando Rebelo. Universidade de Coimbra. pp 385-402.

MARTINS, A. (2004) - "As bacias sedimentares do Baixo Tejo e do Sado". In: FEIO, M. e DAVEAU, S. (Orgs.) - O Relevo de Portugal: Grandes Unidades Regionais. Associação Portuguesa de Geomorfólogos, Coimbra, pp. 49-60.

MEYER, A. e MARTíNEZ-CASASNOVAS, J. A. (1999) - "Prediction of existing gully erosion in vineyard parcels of the NE Spain: a logistic modelling approach". Soil \& Tillage Research, 50, pp. 319-331.

OLAYA, V. (2009) - "Basic Land-Surface Parameters". In: HENGL, T. e REUTER, H. (Eds.) - Geomorphometry: Concepts, Software, Applications. Elsevier, pp. 141 169.

PARKNER, T.; PAGE, M.; MARUTANI, T. e TRUSTRUM, N. (2006) "Development and controlling factors of gullies and gully complexes. East Coast, New Zealand". Earth Surface Processes and Landforms, 31, pp. 187-199.

PERROY, R. L.; BOOKHAGEN, B.; ASNER, G. P. e CHADWICK, O. A. (2010) - "Comparison of gully erosion estimates using airborne and ground based LiDAR on Santa Cruz Island, California". Geomorphology, 118, pp. 288-300.

SUMMERFIELD, M. (1991) - Global Geomorphology. Pearson Education, $537 \mathrm{p}$.

VANDEKERKHOVE, L.; POESEN, J.; OOSTWOUD WIJDENES, D.; GYSSELS, G.; BEUSELINK, L. e DE LUNA, E. (2000) "Characteristics and controlling factors of bank gullies in two semi-arid Mediterranean environments". Geomorphology, 33, pp. 37-58.

VANWALLEGHEM, T.; BORK, H. R.; POESEN, J.; SCHMIDTCHEN, G.; DOTTERWEICH, M.; NACHTERGAELE, J.; BORK, H.; DECKERS, J.; BRÜSCH, B.; BUNGENEERS, J. e DE BIE, M. (2005) - "Rapid development and infilling of a buried gully under cropland, central Belgium”. Catena 63, pp. 221-243.

WELLS, S. G. e GUTIERREZ, A. A. (1982) - "Quaternary evolution of badlands in the south-eastern Colorado plateau, USA". In: BRYAN, R. e YAIR, A. (Eds.) - Badland Geomorphology and Piping. GeoBooks. pp. 239-258.

ZBYSZEWSKI, G. e VEIGA FERREIRA, O. da (1979) - Carta Geológica de Portugal na escala de 1/50000. Notícia explicativa da folha 31-B (Chouto). Serviços Geológicos de Portugal, Lisboa. 